\title{
A case of spinal cord infarction caused by polycythemia vera
}

\author{
J Lee, Y-M Lim and K-K Kim
}

Study design: This is a case report.

Objective: The objective of this study was to report on a 66-year-old woman with a confirmed diagnosis of polycythemia vera who presented with acute spinal cord infarction.

Setting: A 66-year-old woman was previously diagnosed with polycythemia vera and presented with acute paraparesis and urinary retention.

Results: The patient's platelet count was 847000 platelets per $\mu$ l. T2- and diffusion-weighted magnetic resonance imaging revealed hyperintensity at the T12-L1 spinal cord. Computed tomography of the abdominal aorta further revealed multiple thrombi filling the aortic lumen.

Conclusions: Polycythemia vera creates a high risk of systemic thrombosis due to hyperviscosity and platelet activation. Although acute infarction in the spinal cord is a rare complication of this myeloproliferative disease, it should be considered in all affected patients.

Spinal Cord (2015) 53, S19-S21; doi:10.1038/sc.2014.222

\section{INTRODUCTION}

Spinal cord infarction is a rare cause of acute myelopathy, and it is far less common than cerebral infarction. ${ }^{1,2}$ Major causes of spinal cord infarction include therapeutic embolization and atherosclerotic diseases affecting the aorta. ${ }^{1}$ Myeloproliferative disorders, such as polycythemia vera, are a rare cause of acute spinal cord infarction. Here, we report a case of polycythemia vera presenting with an acute spinal cord infarct.

\section{CASE REPORT}

A 66-year-old woman visited our center with acute paraparesis and urinary retention. Three years earlier, she developed a compression fracture at the T12 vertebral body and received sclerotherapy. She had no neurological deficit related to this compression fracture or sclerotherapy. She developed transient paraparesis 3 days before her hospital visit. Her symptoms spontaneously resolved within $5 \mathrm{~h}$, but reoccurred 3 days later. Neurologic examination revealed a symmetric Medical Research Council (MRC) grade 2 weakness in both lower extremities. The patient had transient pain and hyperesthesia in both lower legs, but this soon disappeared and her sensory function was normal. Deep tendon reflex was absent, and there were no upper motor neuron signs. Acute thoracic myelopathy was suspected, such as Adamkiewicz artery thrombosis or segmental thoracic dural arteriovenous malformation-induced hemorrhage. A spinal cord magnetic resonance imaging showed T12-L1 level swelling with T2 high signal intensity in the central portion of the cord. High-signal intensity on diffusion-weighted image with a decreased apparent diffusion coefficient value suggested an acute infarct (Figure 1).

This patient was previously diagnosed with polycythemia vera and Janus kinase 2 mutation. However, she did not receive any treatment after diagnosis. At the time of her revisit to our hospital, her platelet count was 847000 platelets per $\mu \mathrm{l}$ and her hemoglobin level was $16.9 \mathrm{~g} \mathrm{dl}^{-1}$. Her erythropoietin level was below normal. An abdominal computed tomography scan was performed to rule out aortic disease, which revealed multifocal wandering thrombi that were attached to the lumen of the distal aortic arch, descending aorta, abdominal aorta and right common iliac artery (Figure 2). We empirically commenced treatment with antiplatelet and cytotoxic agents.

\section{DISCUSSION}

We report a patient with a prior diagnosis of polycythemia vera who presented with spinal cord infarction and multiple aortic thrombi. Although it is a rare hematologic disease, polycythemia vera needs to be considered as a possible cause of ischemic events in the spinal cord when more frequent etiologies are excluded. Polycythemia vera can also cause recurrent ischemic events, as seen in our current patient. ${ }^{2}$

The diagnosis of polycythemia vera entered a new era with the identification of the Janus kinase 2 mutation in $2005 .{ }^{3}$ The diagnostic criteria for polycythemia vera were revised by the World Health Organization (WHO) in $2008 .^{3}$ It is well established that polycythemia vera is associated with a higher risk of both arterial and venous thrombosis. ${ }^{2}$ This disorder may also cause myocardial infarction, splenic infarction, cerebral infarction and other systemic thromboembolisms. Polycythemia vera also rarely causes aortic thrombus. ${ }^{4}$ The current literature indicates that relatively common causes of paraparesis in polycythemia vera patients is extramedullary hematopoiesis in the spinal epidural space. ${ }^{5}$ However, spinal cord infarction should be considered if polycythemia vera is diagnosed.

To the best of our knowledge, our current patient is the first reported case of spinal cord infarction with polycythemia vera and 

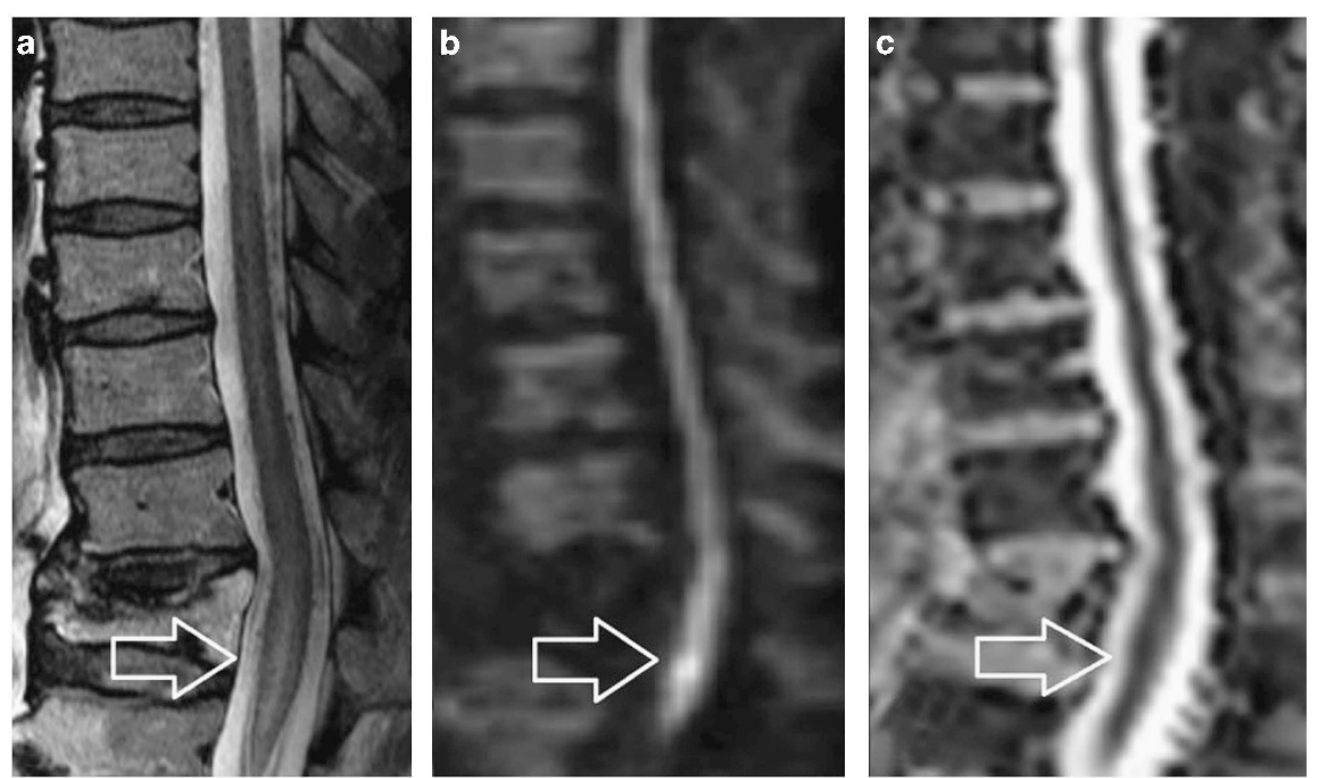

Figure 1 Spinal cord magnetic resonance imaging. (a) T2-weighted image showing hyperintensity in the central spinal cord at the T12-L1 level. The T12 vertebral body shows an old compression fracture. (b, c) Diffusion high signal changes with decreased apparent diffusion coefficient (ADC) values at the T12L1 level.

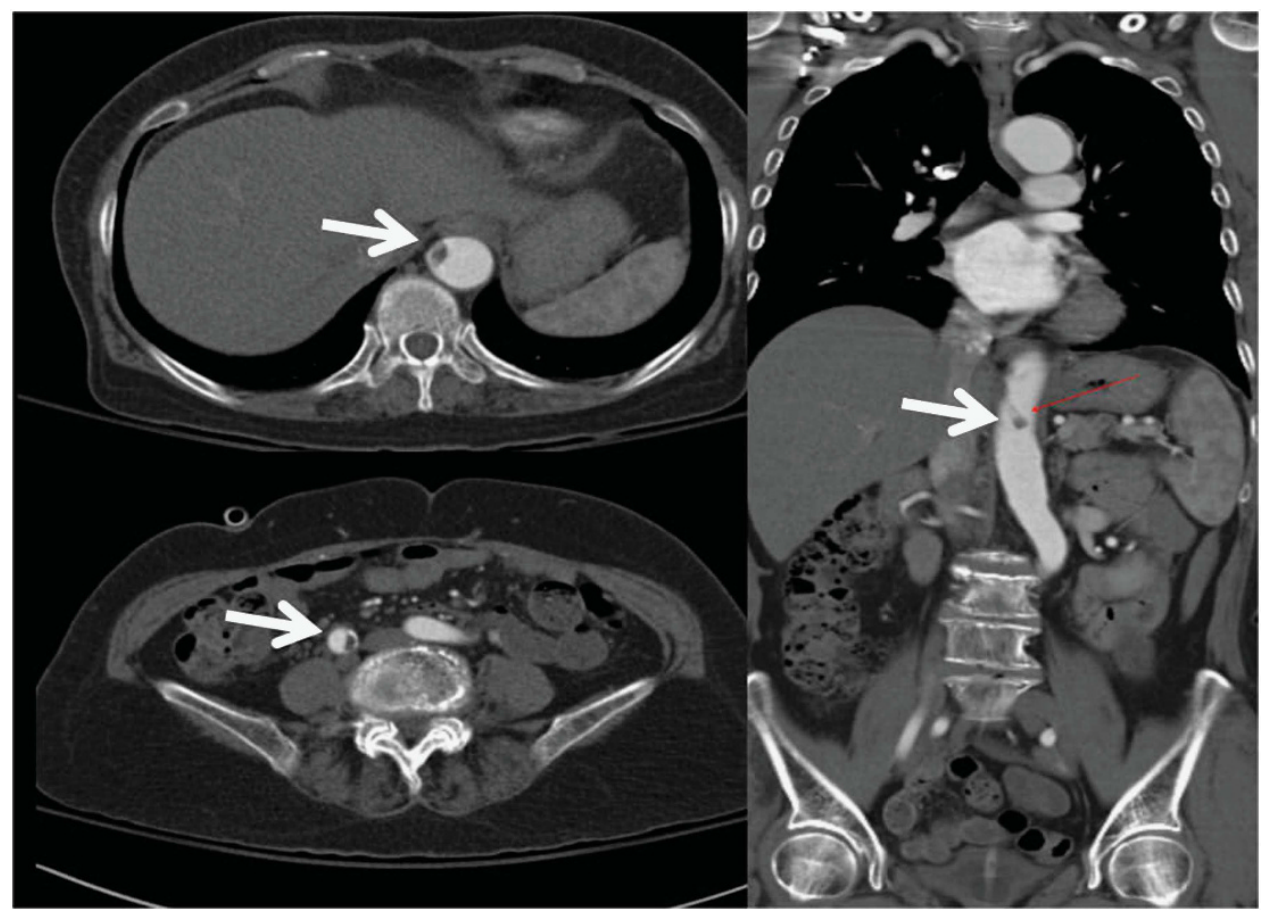

Figure 2 Abdominal computed tomography. Multifocal low-density lesions are shown that are partially attached to the lumen in the distal aortic arch, descending aorta, abdominal aorta and right common iliac artery.

multiple aortic thrombi. It is well known that patients with a spinal cord infarction should receive an evaluation of the abdominal aorta. Common findings include dissection and atherosclerosis, but in our current case we found multiple wandering thrombi in the whole lumen of the aorta. In addition, long-term follow-up and treatments, including the use of antithrombotic agents, are important for preventing relapse. Although there is lack of evidence regarding the secondary prevention of spinal cord infarction by administering lowdose aspirin, this can reduce the risk of thrombosis without increasing the risk of major bleeding in patients with polycythemia vera. ${ }^{6}$

\section{CONFLICT OF INTEREST}

The authors declare no conflict of interest. 
1 Rubin MN, Rabinstein AA. Vascular diseases of the spinal cord. Neurol Clin 2013; 31 : 153-181.

2 Costa S, Marques J, Barradas A, Valverde A. Transient spinal cord ischemia as presenting manifestation of polycythemia vera. Case Rep Neurol 2011; 3: 284-288.

3 Tefferi A. Polycythemia vera and essential thrombocythemia: 2012 update on diagnosis, risk stratification, and management. Am J Hematol 2012; 87: 285-293.
4 Josephson GD, Tiefenbrun J, Harvey J. Thrombosis of the descending thoracic aorta: a case report. Surgery 1993; 114: 598-600.

5 Scott IC, Poynton $\mathrm{CH}$. Polycythaemia rubra vera and myelofibrosis with spinal cord compression. J Clin Pathol 2008; 61: 681-683.

6 Landolfi R, Marchioli R. European Collaboration on Low-dose Aspirin in Polycythemia Vera (ECLAP): a randomized trial. Semin Thromb Hemost 1997; 23: 473-478. 